\title{
A reflection on cupping therapy and historical medical dominance
}

\section{Introduction}

Cupping is a treatment procedure where cups are placed on the skin and then heated to create a vacuum. The pressure from the suction pulls the dermis and muscle tissues into the cup creating petechiae or in Chinese medicine, sha. Drawing the blood, under the skin's surface, to the surface, promotes healthy blood circulation and can be used to treat a range of conditions from common colds to sore muscles. Cupping therapy is a household remedy in many places throughout the world and is recently gaining scientific interest within mainstream medicine.

\section{History of Cupping}

Throughout history, cupping techniques and styles have often resembled the geographic locations they were practiced in, as well as utilizing a region's local materials: animal horns, bamboo, ceramic, glass, metal, and plastic have all been used in this practice found in Ancient Egyptian, Chinese, Unani, Korean, Tibetan, and Latin American cultures, all of which have served the purpose of supporting the body's ability to heal itself. In North Africa, cupping therapy was first documented on Eber's papyrus (1550 BCE), where a cup is the Egyptian glyph to reference a physician. ${ }^{1}$ In Asia, during the Jin dynasty, Ge Hong (281-341 CE) mentions the use of animal horns as a means of draining fluids from the body. ${ }^{1}$ In Arabic and Islamic countries, cups (Hejama) are recommended in the Al-Qanun Fi'lTibb, Canon of Medicine (1025CE), to treat menstrual conditions. ${ }^{1}$

In Chinese medicine, cupping and other similar therapies follow the Daoist model of holism. Holism is the philosophy that systems and their properties must be viewed together, not just as a collection of parts. Daoists contended that no single being or human could exist unless they are seen in relation to nature, as an extension of the universe and as such are impacted by natural phenomenon, such as the seasons and climate, as well as by internal states, such as emotional stress. Disease, according to this concept, is the result of climate, emotions, and/or trauma that create imbalance in the body (See figure One).

Since Chinese medicinal researchers focus on observable principles of balance examined in living bodies, their traditional medicine practices are considered "alternative" by the dominant medical systems, despite having been practiced for centuries in cultures and countries around the world. Alternative medicine is defined as "the promotion or use of practices which are unproven, disproven, impossible to prove, or excessively harmful in relation to their effect." Unfortunately, medicine, particularly Western allopathic medicine, has been viewed solely from an epistemological framework. This framework establishes a theory of knowledge that distinguishes justified belief from opinion. Therefore, evidence-based medicine has been the adopted mode used, predominantly relying on anatomical dissection over any other form of inference or methodology in determining a diagnosis and/or treatment (Table 1).

\section{Discredit the 'alternative'}

But are dissections the best methodology in determining or

\author{
Volume 12 Issue 2 - 2019
}

\author{
Anna Marie Dinall \\ Oregon College of Oriental Medicine, USA \\ Correspondence: Anna Marie Dinall, Oregon College of \\ Oriental Medicine, USA, Tel 505-280-4670, \\ Email ana.dinallo@ocom.edu \\ Received: January 20, 2019 | Published: March 08, 2019
}

proving the effectiveness of therapies such as cupping? Anatomical dissections practices found in mainstream medical societies have actively worked to discredit the 'alternative medicine' of non-white cultures, cultures that did not rely on human anatomy to make medical diagnoses. It was believed by many medical scientists in the 1800's and early 1900's if you did not participate in cutting a body open, then you could not understand human physiology. ${ }^{2}$ Chinese Medicine relies on examination through palpitation, observation, sight, smell, and sound: senses that aren't present in a corpse during autopsy. Thus, for cupping therapy, which has a longstanding history in a Taoist framework, dissection cannot take into account how seasons and climate, as well as internal states, such as emotional stress, impact pathology.

Western medical research and education have been infatuated with understanding disease through dissection. Human dissection in western society is coupled with the omission of cupping therapy as part of standard medical curriculum, despite its popularity in European historical societies. In 1540, Henry VIII of England approved the Charter for the Company of Barber Surgeons. This charter led to the fragmentation of once unified healthcare options that were often provided by women who offered 'alternative medicine'. ${ }^{3}$ In addition, the charter promoted the development of medical specialties and set the stage for scientific dissections in subsequent centuries (Brooke, 2007). Some key European historical discoveries included anatomical dissections of Andreas Vesalius, published in (1543), that challenged European fallacies regarding the relationship between structure and function of the human physiology. ${ }^{2}$ In 1628, William Harvey wrote a book, On the Motion of the Heart, in which he conducted experiments on the circulation of blood flow. ${ }^{2}$ Despite medical advancements, elite aristocrats were the primary beneficiaries of these insights, and thus medical advancements pushed 'alternative' or folk medicine practices like cupping treatment to the periphery.

\section{Fragmentation $\&$ the placebo effect}

To understand why cupping therapy is deemed ineffective it is important to understand the development of the concept of evidence in western medical institutions, and how obtaining evidence has had questionable and corrupt implications. Evidence, according to the Oxford English Dictionary, "is the available body of information" or that which "indicates whether a belief or proposition is true or valid" ${ }^{4}$ Unfortunately, evidence is often situated in epistemology and conditions that assume truth without taking into account cross-cultural 
practices and histories, relying on a single method of dissection rather than using multiple reference points.

In 1835, the first French professorship for medicine was awarded to Jean Cruveilhier. The United States followed. By 1848, 25 of the 40 medical schools in the United States began offering instruction in dissection. Problems arose due to a limited supply of bodies and legal mechanism to obtain anatomical material. Dissection had become acceptable to academics and medical students. As a result, medical schools were located near graveyards:

The new occupation of 'resurrection man' emerged. Fabled in song and story, it satisfied the growing market for fresh bodies with the newly buried corpse of private citizens. The inevitable happened, murder for the possession of corpses. Unknown numbers of disadvantaged citizens may have been killed to this end. ${ }^{2}$

Murdering the living for research was indicative of the power that medical dissection and physiology had over all other types of medical discoveries. ${ }^{2}$ Moreover, the power of dissection was supported by the notion of fragmentation. Rene Descartes (15961650), an influential philosopher, suggested that the mind and body were separate substances, that the mind was distinct from matter Descartes' separation of mind and body created a justification to dissect and dehumanize corpses at an astounding rate. Similarly, Christian religious figures promoted the notion of the body bearing original sin and promoted a separation of body and spirit (Stargrove, personal communication 2018, October 2nd). This fragmentation had serious consequences for the lives lost at the hands of unjust medical doctors all for the sake of obtaining evidence. ${ }^{2}$

In Trick or Treatment, authors Ernst and Singh claimed that cupping therapy lacked "evidence" (2008). They received positive reviews from the scientific community for their publication (2010). The New England Journal of Medicine and Nature gave glowing reviews for Ernst and Singh's call for strict regulation of 'complementary and alternative' medicine. Ernst and Singh reviewed various alternative practices, ranging from acupuncture and cupping therapy, to homeopathy. With minimal investigation, the authors concluded that most if not all alternative practices deceived the general public, with the exception of acupuncture, in treating nauseas and acute pain conditions (Ernst \& Singh, 2010). Perceived benefits of alternative therapy were explained only by dynamics of therapeutic processes or the placebo effect. ${ }^{5}$

The cupping procedure and its visible aspects are likely to generate an above average placebo response.... Cupping has a long history but there is no evidence that it generates positive effects in any medical conditions. ${ }^{5}$

The term "placebo" is often used to degrade the immeasurable attributes of traditional medicine and living organisms. The placebo is a "powerful influence of meaningful symbols in the healing ritual that goes beyond a merely positivistic approach" (p. 1, Jaminson). A positivist framework is the backdrop to the scientific method and validates knowledge through rationalism, experimental designs, and objectivity. In a positivist model of confirming or disproving a hypothesis, it appears that all variables in the natural world and its spiritual phenomenon can be accounted for and controlled. If a research design demonstrates a placebo effect, it is considered defective. Trick or Treatment authors explain human conditioning as one of several mechanisms for how the placebo effect works:

So, if the placebo effect in humans is also a conditioned response, then the explanation for effectiveness would be that a patient simply associates getting better with, for example, seeing a doctor or taking a pill. After all, ever since childhood a patient will have visited a doctor received a pill and felt better. Hence, if a doctor prescribes a pill containing no active ingredient, a so-called sugar pill, then the patient might still experience a benefit due to conditioning. (2008, p. 61).

Conditioning studies have been performed primarily on rats and guinea pigs. ${ }^{5}$ Yet, the authors fail to connect that humans can't be reduced to rats, or vice versa. Moreover, placebo effects in medical research are often variables that include warm engagement, eye contact, a genuine interest, legitimization of the client's emotions, and education. ${ }^{6}$ Nonspecific treatment variables are considered to be major contributors to the placebo effect. ${ }^{6}$ This is no surprise, given that altering personal beliefs and examining social conditioning factors are tools employed by evidenced based mental health treatment models. Moreover, nonspecific variables are key attributes that show a correlation in the reduction in patient pain perceptions ${ }^{6}$ and promote a humanistic approach to client care supported by holistic practices.

\section{Evidence-based}

'Evidence-based' has become a common term for insurance companies, as well as for providers. Evidence- based means that if a researcher can't demonstrate efficacy, the medical insurance companies will not compensate the provider. It also means that medical doctors and allied professionals can recommend only therapies that have earned the legitimacy of being evidence-based. This becomes dangerous when medical scientists and scientific journals support inaccurate information related to alternative practices. Moreover, if cupping therapy and other practices are not considered evidencebased, then medical doctors are not educated in how effective these low cost natural therapies actually are. In this way, evidence, though not backed up, is used to paint an ill and inaccurate portrait of traditional cupping therapy.

Only some 'alternative' medicine practices are covered by insurance, including acupuncture, chiropractic work and medical massage. Traditional medical coverage varies largely by state and often omits natural parallel services used by folk medicine providers. Despite available bodies of information from ancient medical texts, such as Al-Qanun Fi'l-Tibb, Canon of Medicine (1025AD), and Ge Hong (281-341AD) (Chirali (2007), as well as qualitative research, mainstream providers and medical researchers continue to remain illinformed.

Since placebos are portrayed in a negative light in medical research, many alternative providers have sought to justify their practices and find "evidence" by conducting experimental designs. The book The Undeniable Facts about Alternative Medicine, published in 2008, inspired several meta-analyses looking at bodies of research within cupping. Cao \& $\mathrm{Luo}^{7}$ searched 135 randomized controlled cupping studies published from 1992-2010 to identify commonly treated diseases, and found successful patient recovery from conditions such as Bell's palsy, disc herniation, and cervical spondylosis as well as respiratory aid from conditions such as chronic cough, dyspnea, and skin conditions including acne, cold sores, and blisters. Cupping techniques examined ranged from wet cupping to dry, running, and stationary cupping. Cao \& Luo's ${ }^{7}$ meta-analyses demonstrated that cupping therapy, when used with parallel Chinese medical treatments such as herbs and acupuncture, performed significantly superior to cupping alone. Adverse effects were not found in any of the studies, yet authors Ernst and Singh did not include this large body of cupping research in their analysis of 'alternative medicine'. Authors Kim et al. conducted their own meta-analysis, evaluating randomized controlled 
studies to assess the effectiveness of dry cupping in pain reduction (Evidence-Based Complementary and Alternative Medicine, 2011). They found seven clinical trials that demonstrated a reduction in low back pain compared to control and placebo groups.

\section{Cupping as illegitimate}

Ernst and Singh suggest that the philosophical framework of positivism is superior to the holistic framework of Daoist medicine. Seasonal and climate observations yield more data than anatomical dissections for the effectiveness of cupping therapy. ${ }^{8}$ This in part contributes to the erasure of cupping therapy as a legitimate medical treatment as dissection isn't the central point of reference. Trick and Treatment authors continue to critique the Daoist framework: "Chinese medicine emerged from a society that rejected human dissection. Unable to look inside the body, the Chinese developed largely imaginary models of human anatomy that were based on the

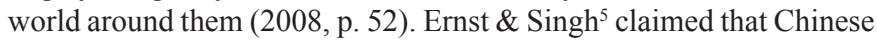
Medicine was flawed or "imaginary." The very gifts of respect for human life, cosmology, and a systemic view of diseases make Chinese medicine holistic. ${ }^{8}$ These same values found in Chinese Medicine are used in many cupping therapy frameworks for traditional medical providers.

Despite evidence published prior to 2008, Ernst and Singh claimed, "The only controlled trial of cupping did not demonstrate this therapy's effectiveness in reducing pain" (p. 307). This is detrimental because it reaffirms the prejudice of traditional medicine as lacking evidence and that it is a mere placebo. Ernst and Singh's statement is dependent upon an epistemological framework. Evidence is most trustworthy when evaluated through various reference points, ${ }^{9}$ over time, and across cultures. For instance, in qualitative research, triangulation is used to limit bias. Triangulation allows the researcher to consult multiple sources, such as archival records, individual narratives, and databases. ${ }^{9}$ Multiple vantage points are used to offer rich, historical, descriptive, in-depth, pattern-oriented, and culturally relevant data. ${ }^{9}$ In quantitative frameworks, data is considered reliable only when it's validated through objective, sterile conditions that create replicable data. ${ }^{9}$ Therefore, a balanced and holistic style of therapy, such as cupping, would not fit into this quantitative framework that dissection and evidence-based medicine promotes.

\section{Conclusion}

Cupping therapy is a component of traditional medicine that has been expressed in many ancient cultures including Egyptian, Arabic, Jewish, Mexican and Asian societies. Despite its well established traditions for alleviating muscular pain, common cold relief and circulatory benefits, it is portrayed as the 'alternative' treatment option with no evidence. Over many decades, Cao \& Luo's ${ }^{7}$ and Kim et al.' ${ }^{10}$ meta-analysis studies demonstrate the effectiveness of dry cupping in pain reduction. Still, unreliable and poorly validated claims exist (Ernst \& Singh, 2010). The term placebo is used to discredit attributes of traditional and natural medicine. Historically, medical boards have discredited folk healthcare because it did not employ human dissection. ${ }^{1}$ Yet, ill-informed scientists continue to promote inaccurate information that discredits and minimizes the benefits of cupping therapy and Chinese Medicine. Their work has been praised in numerous journals and remains largely unchallenged. Only if we dissect these unjustified claims and divorce ourselves from the dominant medical narrative, can we understand why natural practices such as cupping therapy have been marginalized. ${ }^{11}$

\section{Acknowledgments}

None.

\section{Conflicts of interest}

Author declares there are no conflicts of interest.

\section{References}

1. Chirali I Z. Traditional Chinese medicine: cupping therapy. Elsevier Health Sciences.1999.

2. Duffin J. History of medicine: a scandalously short introduction. USA: University of Toronto Press; 2010.

3. Brooke E. Medicine women: A pictorial history of women healers. Quest Books: Theosophical Publishing House; 1997. p. 127.

4. Stargrove M. Personal communication. Class lecture at Oregon College of Oriental Medicine. 2018.

5. Singh S, Ernst E. Trick or Treatment: The undeniable facts about alternative medicine. USA: WW Norton \& Co; 2008.

6. Jamison RN. Nonspecific treatment effects in pain medicine. Pain Clinical Updates. 2011;19(2):1-7.

7. Cao H, Lim X, Liu J. An Updated Review of the Efficacy of Cupping Therapy. Plos One. 2012;7(2):e31793.

8. Sakatani K. Concept of mind and brain in traditional Chinese medicine. Data Science Journal. 2007;6:S220-S224.

9. Patton MQ. Qualitative evaluation and research methods. $2^{\text {nd }}$ end, Newbury Park, CA: SAGE Publications Inc; 1991. p. 532.

10. Kim JI1, Lee MS, Lee DH, et al. Cupping for treating pain: a systematic review. Evid Based Complement Alternat Med. 2011;2011:467014.

11. Conrad R. The hardest thing to see is what is in front of your eyes-quo vadis placebo analgesia? J Pain Res. 2016;9:819-823. 